\title{
$A$ refinement of the Conley index and an application to the stability of hyperbolic invariant sets
}

\author{
ANDREAS FLOER \\ Institut für Mathematik der Ruhr-Universität Bochum, Universitätsstraße 150, D-4630 \\ Bochum, West Germany
}

(Received 11 March 1985 and revised 21 April 1986)

\begin{abstract}
A compact and isolated invariant set of a continuous flow possesses a so called Conley index, which is the homotopy type of a pointed compact space. For this index a well known continuation property holds true. Our aim is to prove in this context a continuation theorem for the invariant set itself, using an additional structure. This refinement of Conley's index theory will then be used to prove a global and topological continuation-theorem for normally hyperbolic invariant sets.
\end{abstract}

\section{Introduction}

The homotopy index theory of $\mathrm{C}$. Conley has proved to be a useful tool in the investigation of qualitative properties of nonlinear problems. Postponing the technical definitions to the next section we recall that to any isolated invariant set $S$ of a continuous flow on $\Gamma$ one can associate an index $I(S)$, which is the homotopy type of a pointed space $X / A$. Here the compact pair $(X, A)$ is an arbitrary index pair for $S, X$ being an isolating neighbourhood of $S$ and $A$ being the exit set, see [2] and [3]. One of the crucial and most useful properties of the homotopy index is that it is invariant under continuation, similar to the invariance of the Leray Schauder index.

In particular, if $S_{1}$ and $S_{2}$ are related by continuation, then $I\left(S_{1}\right)=I\left(S_{2}\right)$ and there is an isomorphism $j$ of the Alexander cohomology

$$
j: H^{*}\left(X_{1}, A_{1}\right) \rightarrow H^{*}\left(X_{2}, A_{2}\right)
$$

of the corresponding index pairs for $S_{1}$ and $S_{2}$, respectively. The topology of the invariant set $S$ is, however, in general not an invariant under continuation. In fact, even if $I(S)$ is very complicated, one cannot conclude that $S$ contains more than one point.

It is the aim of this note to show that an additional structure, which actually occurs in applications, can be used to define an additional invariant of $S$ under continuation, which carries along some topology of $S$ itself. To be more precise, the additional structure is a continuous map $\alpha: \Gamma \rightarrow T$ into a topological space $T$. Using this map one defines an operation of the cohomology ring $H^{*}(T)$ on $H^{*}(X, A)$ by

$$
*_{\alpha}: H^{*}(T) \times H^{*}(X, A) \rightarrow H^{*}(X, A):(v, u) \rightarrow\left(\left.\alpha\right|_{X}\right)^{*} v \cup u
$$


Here, $\left.\alpha\right|_{X}: X \rightarrow T$ is the restriction of $\alpha$ and $\cup: H^{*}(X) \otimes H^{*}(X, A) \rightarrow H^{*}(X, A)$ is the cup product. The first result is that this $H^{*}(T)$-module structure of $H^{*}(X, A)$ is an invariant of $S$ under continuation.

We shall apply this construction to prove a continuation result for a normally hyperbolic invariant manifold $S$ in the special case where $S$ is a retract of $\Gamma$. In fact, as long as the hypothesis of the continuation invariance of the homotopy index is preserved, the cohomology of $S$ will be shown to be stable in a certain sense. It is of course well known that under appropriate conditions, see [5] and [9], a normally hyperbolic invariant manifold of a differentiable flow persists under small and smooth perturbations. In contrast to this we prove a global continuation theorem, giving up, of course, the differentiable structure of the invariant set. The motivation for the above invariant in C. Conley's index theory comes from our proof of the Arnold conjecture for fixed points of symplectic maps on surfaces of higher genus, see [6] and [7]. It is used there to find critical points of the Hamiltonian action functional on the loop space. The critical points are the solutions of the exact Hamiltonian equations on the surface. In this application, the map $\alpha$ maps the loop space onto the surface and is used to prove that the union of the bounded trajectories of the gradient flow on the loop space inherits the topology of the surface.

I would like to thank C. Conley and E. Zehnder for valuable discussions. This work was supported by the Stiftung Volkswagenwerk.

\section{Statement of results}

Recall that a flow on a Hausdorff space $\Gamma$ is a continuous map

$$
\chi: \Gamma \times \mathbb{R}_{+} \supset U_{\Gamma} \rightarrow \Gamma:(x, t) \rightarrow \chi(x, t)=x \cdot t,
$$

where $U_{\Gamma}$ is a neighbourhood of $\Gamma \times\{0\}$ in $\Gamma \times \mathbb{R}_{+}$with the following property: If $(x, t)$ and $(x \cdot t, s) \in U_{\Gamma}$, then $(x, t+s) \in U_{\Gamma}$ and

$$
(x \cdot t) \cdot s=x \cdot(t+s) \text {. }
$$

For example, every Lipschitz continuous vector field on a differentiable manifold $\boldsymbol{M}$ induces a flow on $\boldsymbol{M}$. In some applications, a flow is equivariant with respect to a topological group $G$, i.e. there is a continuous operation $G \times \Gamma \rightarrow \Gamma:(g, \gamma) \rightarrow g \gamma$ so that for $(\gamma, t) \in U_{\Gamma}$ we have $(g \gamma, t) \in U_{\Gamma}$ and $g(\gamma \cdot t)=(g \gamma) \cdot t$. We will always assume that $G$ is compact. A subset $X$ of $\Gamma$ is called $G$-invariant if $g \in G$ and $\gamma \in X$ implies $g \gamma \in X$. We call it invariant or flow invariant, if for all $(\gamma, t) \in U_{\Gamma}$ with $\gamma \in X$ we have $\gamma \cdot t \in X$. A map $f: X \rightarrow Y$ is called equivariant if $f(g \gamma)=g(f(\gamma))$.

Definition 1. For any ( $G$-invariant) subset $U$ of $\Gamma$, define the maximal invariant set in $S(U)$ by

$$
\begin{aligned}
U^{\infty} & :=\bigcap_{t \geq 0} U \cdot t \\
U^{-\infty} & :=\left\{\gamma \in U \mid \gamma \cdot t \in U \text { for all } t \in \mathbb{R}_{+}\right\} . \\
S(U) & =U^{\infty} \cap U^{-\infty} .
\end{aligned}
$$

For any subset $A$ of $\Gamma$, let $\bar{A}$ denote the closure and $\AA$ the interior. We call $U$ isolating if $\overline{S(U)} \subset \stackrel{\circ}{U}$. An invariant (and $G$-invariant) set $S$ is called an isolated 
invariant set if there exists a neighbourhood $U$ of $S$ so that $S=S(U)$. In this case, $U$ will be called an isolating neighbourhood of $S$.

In the following, we will restrict our attention to compact isolated invariant sets of flows on locally compact spaces $\Gamma$. In this case, Conley [2] defines an invariant for $S$ in the following way.

Definition 2. Let $S$ be a compact isolated invariant set of the flow $\chi$ on $\Gamma$. An index pair for $S$ is a compact pair $A \subset X$ in $\Gamma$ so that

(1) $X-A$ is an isolating neighbourhood of $S$;

(2) $A$ is positively invariant with respect to $X$, i.e. if $\gamma \in A$ and $\gamma \cdot[0, t] \subset X$ for $t \geq 0$, then $\gamma \cdot[0, t] \subset A$.

(3) If $\gamma \in X$ and $\gamma \cdot t \notin X$ for some $t>0$, then there exists $s \in[0, t]$ so that $z \cdot s \in A$. In [2], the homotopy index $I(S)$ is defined as the homotopy type of the quotient $X / A$. In this paper, we will consider the cohomological index $I^{*}(S)$ defined by

$$
I^{*}(S)=H^{*}(X, A) \text {. }
$$

Here, $H^{*}(X, A)$ are the (G-equivariant) Alexander cohomology groups of the pair $(X, A)$ with coefficients in some ring $R$ (see [10] and $\S 3$ below for the equivariant case). It is proved in [2] that $I(S)$ does not depend on the choice of the index pair but only on the flow and on the set $S$. The main point of Conley's index theory is that it is also invariant under certain deformations of the flow, which we will describe next.

Let $\Lambda$ be some Hausdorff space and let $\chi_{\lambda}, \lambda \in \Lambda$, be a family of local flows on $\Gamma$ so that

$$
\chi: \Lambda \times \Gamma \times \mathbb{R}_{+} \ni(\lambda, \gamma, t) \rightarrow\left(\lambda, \chi_{\lambda}(t, \gamma)\right)
$$

is a flow on $\Lambda \times \Gamma$. Such a flow will be called a product flow. Following [2, IV], we define

Definition 3. A (G-invariant) parametrization $p$ of a flow $\chi$ on $\Gamma$ is a $\chi$-invariant (and $G$-equivariant) map $p: \Gamma \rightarrow \Lambda$, where $\Lambda$ is a Hausdorff space and $G$ operates trivially on $\Lambda$, i.e. $g \lambda=\lambda$ for all $g \in G$ and $\lambda \in \Lambda$. We call $p$ a local product parametrization if for every $\gamma \in \Gamma$ there exists an open neighbourhood $U$ which is equivalent to a local product flow with parameter set $p(U)$. For any parametrized local flow $(\chi, p)$ and $\lambda \in \Lambda$, let $\chi_{\lambda}$ denote the flow restricted to the leaf $\Gamma_{\lambda}:=p^{-1}[\lambda]$. As usual, two ( $G$-equivariant) flows $\chi_{i}$ on $\Gamma_{i}, i=1,2$, are called equivalent if there exists a (G-equivariant) homeomorphism $h: \Gamma_{1} \rightarrow \Gamma_{2}$ so that $\chi_{2}^{t} \circ h=h \circ \chi_{1}^{t}$. Now consider the set

$$
\mathscr{S}=\left\{(\lambda, S) \mid \lambda \in \Lambda \text { and } S \text { is an isolated invariant set of } \chi_{\lambda}\right\} .
$$

On $\mathscr{S}$ define the topology generated by the sets

$$
\theta_{U}=\left\{S\left(U \cap \Gamma_{\lambda}\right) \mid \lambda \in \Lambda \text { and } U \cap \Gamma_{\lambda} \text { is isolating in } \Gamma_{\lambda}\right\},
$$

where $U$ is any open ( $G$-invariant) subset of $\Gamma$. Now we call two elements $S$ and $S^{\prime}$ of $\mathscr{S}$ related by continuation if they can be connected by a continuous path in $\mathscr{S}$. In this case it follows from [2] that $I(S)$ is equal to $I\left(S^{\prime}\right)$. 
Of course, one is interested in the topology of $S$ rather than in $I(S)$. As remarked in the introduction, the topology of $S$ is not in general related to the topology of the homotopy index. As an additional structure, we therefore assume that there is a continuous ( $G$-equivariant) map

$$
\alpha: \Gamma \rightarrow T
$$

where $T$ is a topological space with a continuous operation of $G$. Then we define an operation of $H^{*}(T)$ on $I^{*}(S)=H^{*}(X, A)$ by

$$
H^{*}(T) \times H^{*}(X, A) \rightarrow H^{*}(X, A):(v, u) \rightarrow v{ }_{\alpha} u=\left(\left.\alpha\right|_{X}\right)^{*} v \cup u
$$

Our first result is the following theorem:

THEOREM 1. Let $\chi$ be a continuous (G-equivariant) flow on the locally compact Hausdorff space $\Gamma$ and let $p: \Gamma \rightarrow \Lambda$ be a (G-invariant) local product parametrization. Moreover, let $\alpha: \Gamma \rightarrow T$ be a (G-equivariant) continuous map.

Then for any compact isolated invariant (and $G$-invariant) set $S$ in any leaf $\Gamma_{\lambda}$, $\lambda \in \Lambda$, there exists a (G-invariant) index pair. Moreover, if two compact isolated invariant (and G-invariant) sets $S$ and $S^{\prime}$ in $\Gamma_{\lambda}$ and $\Gamma_{\lambda}$, with index pairs $(X, A)$ and $\left(X^{\prime}, A^{\prime}\right)$, respectively, are connected in $\mathscr{S}$, then there exists an isomorphism $i: H^{*}(X, A) \rightarrow H^{*}\left(X^{\prime}, A^{\prime}\right)$ satisfying

$$
i\left(u *_{\alpha} v\right)=u *_{\alpha} i(v)
$$

In view of theorem 1 , we will from now on regard the module $I^{*}(S)$ of (2.4) as an invariant of $S$ alone. The proof of theorem 1 will be analogous to the proof of the invariance of the homotopy index in [2]. We are in particular interested in a special case. First recall that a subset $X \subset Y$ is a retract if there exists a continuous map $r: Y \rightarrow X$ so that $\left.r\right|_{X}=\mathrm{id}_{X}$. Such a map is called a retraction.

Definition 4. A connected compact isolated invariant (and $G$-invariant) set $S$ of a topological flow on a locally compact space $\Gamma$ is called $*$-hyperbolic of index $n \in \mathbb{N}$ if $S$ is a ( $G$-equivariant) retract of some ( $G$-invariant) neighbourhood $Q$ of $S$ in $\Gamma$ and if there exists $u \in I^{*}(S)$ of dimension $n$ so that the map

$$
H^{*}(S) \rightarrow I^{*}(S): a \rightarrow a * r u
$$

is an isomorphism for any ( $G$-equivariant) retraction $r: Q \rightarrow S$.

An example is described in the following proposition:

Proposition 1. Assume that the flow $\chi$ on the $C^{2}$-manifold $M$ is induced by $a$ (G-invariant) $C^{2}$-vector field $V$. Let $S$ be a compact invariant (and G-invariant) $C^{2}$-submanifold of $M$. Assume moreover that there exists a decomposition

$$
\left.T M\right|_{S}=T S \oplus E^{+} \oplus E^{-}
$$

which is invariant under the covariant linearization $D V: T M \rightarrow T M$ of the vector field $V$ with respect to some ( $G$-invariant) metric $\langle$,$\rangle on M$ so that for some constant $m>0$ :

$$
\begin{aligned}
& \langle\xi, D V \xi\rangle \leq-m\langle\xi, \xi\rangle \quad \text { for } \xi \in E^{-} \\
& \langle\xi, D V \xi\rangle \geq m\langle\xi, \xi\rangle \quad \text { for } \xi \in E^{+} .
\end{aligned}
$$

Then $S$ is a *-hyperbolic invariant set of $\chi$ of index $\operatorname{dim} E^{+}$. 
The proof of this proposition will be given in $\$ 4$. Hyperbolic invariant sets as described in the hypothesis of proposition 1 have certain stability properties with respect to small perturbations of the flow (see, for example, [5]). The main application of theorem 1 is the following stability property of the invariant set $S$.

THEOREM 2. Assume that for some $\lambda \in \Lambda$, the leaf $\Gamma_{\lambda}$ contains a compact ( $G$-invariant) *-hyperbolic invariant set $S$ which is a (G-equivariant) retract of $\Gamma$ by the retraction $\alpha$. Then for every isolated invariant set $S^{\prime}$ in $\Gamma_{\lambda^{\prime}}$ which is related to $S$ by continuation, the homomorphism

$$
\left(\left.\alpha\right|_{S^{\prime}}\right)^{*}: H^{*}(S) \rightarrow H^{*}\left(S^{\prime}\right)
$$

is injective.

Theorem 2 will be proved in $\S 6$. In the general situation of theorem 1 , we can still use the module structure of $I^{*}(S)$ in the following way:

Definition 5. For a compact isolated invariant (and $G$-invariant) set $S$, set

$$
\begin{aligned}
& l_{\alpha}(S):=1+\max \{l \in \mathbb{N} \mid \text { there exist classes } \\
& \qquad \sigma_{1} \cdots \sigma_{l} \in H^{*}(T), \sigma_{i} \notin H^{0}(T), \text { and } u \in I^{*}(S) \\
& \text { so that } \left.\sigma_{1} *_{\alpha} \cdots *_{\alpha} \sigma_{l} *_{\alpha} u \neq 0\right\} .
\end{aligned}
$$

The number $l_{\alpha}(S)$ can be used to estimate the cup length of $S$ :

Proposition 2. There are classes $u_{i} \in H^{*}(S), 1 \leq i \leq l-1, l=l_{\alpha}(S), \operatorname{dim} u_{i}>0$, so that $u_{1} \cup \cdots \cup u_{l-1} \neq 0$. In fact, one can choose $u_{i}=\left.\alpha^{*}\right|_{S} \sigma_{i}$ with $\sigma_{i}$ as in definition 5 .

Proposition 2 is a reformulation of a remark by V. Benci [1]. Its proof is analogous to that of theorem 2 and will therefore be omitted.

\section{G-equivariant Alexander cohomology}

In this section, we recall some well known properties of Alexander cohomology theory (see, for example, [10]) and establish the corresponding statements for equivariant cohomology, following the exposition in [4]. Throughout this section, we assume that $A$ is a closed ( $G$-invariant) subset of a compact space $X$ with $G$ operating continuously on $X$.

We define the equivariant Alexander cohomology of the pair $(X, A)$ as the ordinary Alexander cohomology of the pair $((E \times X) / G,(E \times A) / G)$ where $E$ is a contractible space with a free operation of $G$ (see [4]). $E$ is not a compact space in general, but there exists an approximation of $E$ by $G$-invariant compact subsets $E^{m} \subset E$, $m \in \mathbb{N}$ so that for any pair $(X, A)$ as above, the inclusions

$$
i_{m}:\left(\left(E^{m} \times X\right) / G,\left(E^{m} \times A\right) / G\right) \rightarrow((E \times X) / G,(E \times A) / G)
$$

induce isomorphisms in (Alexander) cohomology in dimensions less than $m$.

We will need the following two properties, which are related to the so-called continuity property of Alexander cohomology.

LEMMA 1. If $\left(X_{t}, A_{t}\right), t \in[0, \infty)$, is a family of (G-invariant) compact pairs with $(X, A)=\bigcap_{t \geq 0}\left(X_{t}, A_{t}\right)$, then the inclusions $i_{t}:(X, A) \rightarrow\left(X_{t}, A_{t}\right)$ induce isomorphisms 
in (G-equivariant) Alexander cohomology in the direct limit for $t \rightarrow \infty$. In particular, if $H^{*}\left(X_{t}, A_{t}\right)=0$ for all $t$, then $H^{*}(X, A)=0$.

Proof. In the non-equivariant case, this lemma is theorem 6 of $[10,6.6]$. The equivariant case can be reduced to this case since

$$
\bigcap_{t \geq 0}\left(E^{m} \times A_{t}\right) / G=\left(E^{m} \times A\right) / G .
$$

LemMA 2. Let $X / A$ denote the quotient of $X$ by $A$, i.e. the topological space where all points of $A$ are identified to one point $\{A\}$. Then the projection map $r:(X, A) \rightarrow$ $(X / A,\{A\})$ induces isomorphisms in (G-equivariant) cohomology.

Proof. For the same reason as in the proof of lemma 1, it suffices to consider the non-equivariant case. Note that $r$ is the composition of maps

$$
(X, A) \stackrel{i}{\rightarrow}(X \cup C A, C A) \stackrel{r}{\rightarrow}(X \cup C A / C A,\{C A\})=(X / A,\{A\})
$$

where $C A$ is the cone over $A$ and $X \cup C A$ is the mapping cone of the inclusion $A \subset X$ (see [10, p. 365]). $i$ induces cohomology isomorphisms by the strong excision theorem $([10$, p. 317$])$ and $r$ induces cohomology isomorphisms by $[10$, theorem $6.9 .15]$.

\section{Proof of theorem 1}

In [2], it is proved that for every isolating neighbourhood $Q$ of $S$ there exists an index pair $(X, A)$ with the additional property that both $X$ and $A$ are positively invariant with respect to $Q$. In this case we call $(X, A)$ an index pair in $Q$. It is also convenient to drop the condition that $A \subset X$ and to write $H^{*}(X, A):=$ $H^{*}(X, A \cap X)$. In the equivariant case, the existence of $G$-invariant index pairs in any $G$-invariant isolating neighbourhood of $S$ follows from

Lemma 3. Assume that $Q$ is a G-invariant isolating neighbourhood of the compact invariant and $G$-invariant set $S$. Then if $(X, A)$ is an index pair in $Q$, then so is $(G X, G A)$, where $G X=\{g \gamma \mid g \in G$ and $\gamma \in X\}$.

Proof. Property (1) in definition 2 holds for $(G X, G A)$ since $G$ is compact. The verification of properties (2) and (3) is left to the reader.

For the rest of this section, in the equivariant case all sets are $G$-invariant and all maps are $G$-equivariant. We say that two compact pairs in $\Gamma$ are $\alpha$-equivalent and write $\left(X_{1}, A_{1}\right) \sim\left(X_{2}, A_{2}\right)$, if there exists a continuous map of pairs $f:\left(X_{1}, A_{1}\right) \rightarrow$ $\left(X_{2}, A_{2}\right)$ inducing isomorphisms $f^{*}: H^{*}\left(X_{2}, A_{2}\right) \rightarrow H^{*}\left(X_{1}, A_{1}\right)$ and satisfying $\left.\left.\alpha\right|_{X_{2}} \circ f \simeq \alpha\right|_{X_{1}}$. In the usual way, this relation generates an equivalence relation for which we will use the same name and notation. Obviously, it suffices to show that the index pairs in the assertion of theorem 1 are $\alpha$-equivalent.

Following [2], we define for any index pair $(X, A)$ for $S$ in $\Gamma$ the compact sets

$$
\begin{aligned}
X^{t} & =\bigcap_{0 \leq \tau \leq t} X \cdot \tau, \\
A^{-t} & =\{\gamma \in X \mid \gamma \cdot[0, t] \cap A \neq \varnothing\} .
\end{aligned}
$$

One can verify that if $(X, A)$ is an index pair for $S$ in $Q$, then so is $\left(X, A^{-t}\right)$. 
LemMA 4. (1) For each neighbourhood $Q^{\prime} \subset Q$ of $S$ there exists an index pair $(X, A)$ in $Q$ so that $\overline{X-A} \subset Q^{\prime}$ and $\left(X \cap Q^{\prime}, A \cap Q^{\prime}\right)$ is an index pair in $Q^{\prime}$.

(2) For any two index pairs $(X, A)$ and $(\tilde{X}, \tilde{A})$ in $Q$, there exists $t \geq 0$ so that $\left(X^{t}, A \cap X^{t}\right) \subset\left(\tilde{X}, \tilde{X} \cap \tilde{A}^{-t}\right)$.

(3) The inclusions

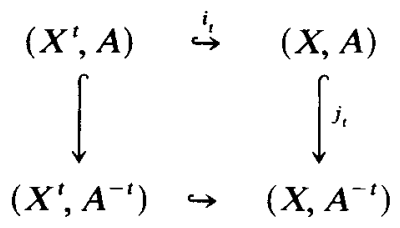

induce isomorphisms in Alexander cohomology and are therefore $\alpha$-equivalences.

Proof. The first two assertions are statements 4.1.D and 4.2.D, respectively, of [2, III]. To prove (3) we follow [2, III.4] and consider the map $f_{t}:(X, A) \rightarrow\left(X^{t} / A\right.$, $\{A\})$ given by $f_{t}(\gamma)=\gamma \cdot t$ if $\gamma \cdot[0, t] \subset X$ and $f(\gamma)=\{A\}$ otherwise. Let $r:(Y, B) \rightarrow$ $(Y / B,\{B\})$ denote the contracting map. Then there is a homotopy

$$
r \simeq i_{t} \circ f_{t}:(X, A) \rightarrow(X / A,\{A\})
$$

given by the family $\left(i_{\tau} \circ f_{\tau}\right)_{\tau \in[0, t]}$. Moreover, a homotopy

$$
r \simeq f_{t} \circ i_{t}:\left(X^{t}, A\right) \rightarrow\left(X^{t} / A,\{A\}\right)
$$

is given by the family of maps $\left(f_{\tau} \circ i_{t}\right)_{\tau \in[0, t]}$. (Note that $f_{\tau}$ preserves $X^{t}$ for $\tau \leq t$ ). Obviously, all these maps are $G$-equivariant whenever the flow is. Hence in ( $G$ equivariant) cohomology, we have $f_{t}^{*} i_{t}^{*}=r^{*}$ and $i_{t}^{*} f_{t}^{*}=r^{*}$, and by lemma $2, i_{t}^{*}$ has a two sided inverse.

In the same way, one shows that $j_{t}$ is an isomorphism, using the map $g_{t}:\left(X, A^{-t}\right) \rightarrow$ $(X / A,\{A\})$ given by $g_{t}(\gamma)=\gamma \cdot t$ for $\gamma \cdot[0, t] \subset X-A$ and $g(x)=\{A\}$ otherwise. Finally, repeating the first argument with $(X, A)$ replaced by the index pair $\left(X, A^{-t}\right)$ proves property (3). For more details, see [2, III.4.2].

From (1) and (3), it follows immediately that any 2 index pairs $(X, A)$ and $(\bar{X}, \bar{A})$ in the same isolating neighbourhood $Q$ are $\alpha$-equivalent by the inclusions $(X, A) \leftarrow$ $\left(X^{t}, A\right) \rightarrow\left(\bar{X}, \bar{A}^{-t}\right) \leftarrow(\bar{X}, \bar{A})$. Moreover, the inclusion $\left(X \cap Q^{\prime}, A \cap Q^{\prime}\right) \rightarrow(X, A)$ in (1) of lemma 2.1 is an $\alpha$-equivalence by excision. Consequently, all index pairs in $Q^{\prime} \subset Q$ are $\alpha$-equivalent to all index pairs in $Q$. If $Q$ and $\bar{Q}$ are two different isolating neighbourhoods, then apply this consideration to $\bar{Q} \supset \bar{Q} \cap Q \subset Q$.

We now prove the continuation invariance. Since we only consider path connected elements in $\mathscr{S}$, we can assume that $\Gamma=\mathbb{R}$. It also suffices to consider a small neighbourhood $U_{\mu}$ of $\mu \in \mathbb{R}$. Moreover, since the parametrization is locally product, and since we can restrict ourselves to a compact subset of $\Gamma$, we can assume that $\chi_{\lambda}, \lambda \in U_{\mu}$, is a continuous family of flows on $\Gamma_{\mu}$. The continuous map $\alpha$ then corresponds to a continuous family of maps $\alpha_{\lambda}: \Gamma \rightarrow T$ in the sense that the map $\Gamma_{\mu} \times U_{\mu} \rightarrow T:(\gamma, \lambda) \rightarrow \alpha_{\lambda}(\gamma)$ is continuous.

Now assume that $Q$ is an isolating neighbourhood of $S=S\left(Q, \chi_{\mu}\right)$ with respect to the flow $\chi_{\mu}$. By [2, IV.1.2.A], there exist two index pairs $(X, A)$ and $\left(X^{\prime}, A^{\prime}\right)$ of $S_{\mu}$ in $Q$ so that

$$
\bar{X} \subset \dot{X}^{\prime} \quad \text { and } \quad \bar{A} \subset \AA^{\prime}
$$


and $\overline{X^{\prime}-A} \subset \dot{Q}$. Define for $\lambda \in U_{\mu}$ the set $A_{\lambda}=P_{\lambda}(A, Q)$ as the intersection of all positively invariant subsets of $Q$ with respect to $\chi_{\lambda}$ which contain $A$. Correspondingly, define $X_{\lambda}=P_{\lambda}(X, Q), A_{\lambda}^{\prime}=P_{\lambda}\left(A^{\prime}, Q\right)$, and $X_{\lambda}^{\prime}=P_{\lambda}\left(X^{\prime}, Q\right)$. Again by [2, IV.1.2.A], there exists a neighbourhood $V_{\mu} \subset U_{\mu}$ of $\mu$ so that for $\lambda \in V_{\mu}$ :

(1) $Q$ is isolating with respect to $\chi_{\lambda}$;

(2) Both $\left(X_{\lambda}, A_{\lambda}\right)$ and $\left(\mathrm{X}_{\lambda}^{\prime}, A_{\lambda}^{\prime}\right)$ are index pairs of $S_{\lambda}:=S\left(Q, \chi_{\lambda}\right)$ in $Q$ with respect to $\chi_{\lambda}$.

Moreover, it follows from (4.3) that we can make $V_{\mu}$ small enough that $\left(X_{\lambda}, A_{\lambda}\right) \subset$ $\left(X_{\lambda}^{\prime}, A_{\lambda}^{\prime}\right)$ for all $\lambda \in V_{\mu}$. Now consider the inclusions

$$
(X, A) \subset\left(X_{\lambda}, A_{\lambda}\right) \subset\left(X^{\prime}, A^{\prime}\right) \subset\left(X_{\lambda}^{\prime}, A_{\lambda}^{\prime}\right) .
$$

Since it was shown above that $(X, A) \sim\left(X^{\prime}, A^{\prime}\right)$ and $\left(X_{\lambda}, A_{\lambda}\right) \sim\left(X_{\lambda}^{\prime}, A_{\lambda}^{\prime}\right)$ as index pairs, and since $\alpha_{\lambda} \simeq \alpha_{\mu}$, this completes the proof of theorem 1 .

\section{The proof of proposition 1}

We construct a 'small' index pair for $S$ which is homotopy equivalent to the Thom complex (compare [10, p. 259]) of $E^{+}$. For every $\varepsilon>0$, define the disc bundles

$$
B_{\varepsilon}^{ \pm}=\left\{(s, \xi) \in E^{ \pm} \mid\|\xi\| \leq \varepsilon\right\}
$$

and the fibrewise product $B_{\varepsilon}=B_{\varepsilon} \times B_{\varepsilon}^{+}$. Consider for $x \in M$ the exponential map $\exp _{x}: T_{x} M \rightarrow M$ defined with respect to the metric $($,$) (see, for example, [8, I, \S 6]$ ). It is well known that for $\varepsilon_{0}$ small enough, the map

$$
\delta: B_{\varepsilon_{0}} \rightarrow M:(s, \xi) \rightarrow \exp _{s} \xi
$$

is a diffeomorphism onto its image. Instead of $V$ we can therefore consider the vector field $W=\left(\delta^{-1}\right)^{*} V$ on $B_{\varepsilon_{0}}$, which induces a flow $\chi_{W}$ equivalent to the original flow $\chi$.

Let $\pi_{ \pm}: \nu(S) \rightarrow E^{ \pm}$be the projections corresponding to the decomposition of $\left.T M\right|_{S}$ and define on $B_{\varepsilon}$ the smooth functions $b_{ \pm}(s, \xi)=\left\|\pi_{ \pm}(s) \xi\right\|^{2}$. Consider the sets

$$
A_{\varepsilon}^{ \pm}=\left\{(s, \xi) \in B_{\varepsilon} \mid b_{ \pm}(s, \xi)=\varepsilon^{2}\right\} .
$$

For every $(s, \xi) \in A_{\varepsilon}^{+}$, we have

$$
\begin{aligned}
\frac{d}{d t}\left(b_{+}\left(\chi_{W}^{t}(s, \xi)\right)\right) & =2\left\langle\pi_{+} \xi, \pi_{+} W(s, \xi)\right\rangle \\
& =2\left\langle\pi_{+} \xi, \pi_{+}\left((D V)(s) \xi+R_{2} W(s, \xi)\right)\right\rangle \\
& \geq 2 m\left\|\pi_{+} \xi\right\|^{2}-\left\|\pi_{+} \xi\right\|\left\|R_{2} W(s, \xi)\right\|
\end{aligned}
$$

where

$$
\begin{aligned}
\left\|R_{2} W(s, \xi)\right\| & \leq\left(\max _{x \in B_{\varepsilon}}\left\|D^{2} W(x)\right\|\right) \cdot\|\xi\|^{2} \\
& \leq C \varepsilon^{2},
\end{aligned}
$$

by Taylor's theorem. Hence there exists an $\varepsilon_{1} \in\left(0, \varepsilon_{0}\right)$ so that for all $\varepsilon \in\left(0, \varepsilon_{1}\right)$ and $(s, \xi) \in A_{\varepsilon}^{+}$:

$$
\frac{d}{d t}\left(b_{+}\left(\chi_{w}^{t}(s, \xi)\right)\right) \geq m \varepsilon^{2}>0
$$


Similarly, we can choose $\varepsilon_{1}$ small enough so that for all $\varepsilon \in\left(0, \varepsilon_{1}\right)$ and $(s, \xi) \in A_{\varepsilon}^{-}$:

$$
\frac{d}{d t}\left(b_{-}\left(\chi_{W}^{t}(s, \xi)\right)\right) \leq-m \varepsilon^{2}<0 .
$$

It follows immediately that for $\varepsilon \in\left(0, \varepsilon_{1}\right), B_{\varepsilon}$ is an isolating neighbourhood for the flow $\chi_{W}$, since every $(s, \xi) \in \partial B_{\varepsilon}=A_{\varepsilon}^{+} \cup A_{\varepsilon}^{-}$leaves $B_{\varepsilon}$ either forwards or backwards in time. Moreover, it is easy to see that $A_{\varepsilon}^{+}$is an exit set for $B_{\varepsilon}$. Since $\bigcap_{0<\varepsilon<\varepsilon_{1}} B_{\varepsilon}=S$, there exists an $\varepsilon \in\left(0, \varepsilon_{1}\right)$ so that $(X, A):=\left(B_{\varepsilon}, A_{\varepsilon}^{+}\right)$is an index pair for $S$. Such an index pair is also called an isolating block.

Any local retraction $\alpha: B_{\varepsilon} \rightarrow S$ is homotopic to the fibre bundle projection $\pi: B_{\varepsilon} \rightarrow S$ by the homotopy $H(\tau,(s, \xi))=\alpha(s, \tau \xi)$. Obviously, $\pi_{+}: B_{\varepsilon} \rightarrow B_{\varepsilon}^{+}$defines a fibre homotopy equivalence $(\boldsymbol{X}, \boldsymbol{A}) \rightarrow\left(B_{\varepsilon}^{+}, \partial B_{\varepsilon}^{+}\right)$. By the Thom isomorphism theorem (see $\left[10\right.$, p. 259]), there exists a class $u \in H^{*}\left(B_{\varepsilon}^{+}, \partial B_{\varepsilon}^{+}\right)$so that the map $H^{*}(S) \rightarrow H^{*}\left(B_{\varepsilon}^{-}, \partial B_{\varepsilon}^{-}\right)$given by $v \rightarrow \alpha^{*} v \cup u$ is an isomorphism. Then for $u_{W}:=$ $\left(\pi_{-}^{*}\right)^{-1} u$, the homomorphism

$$
\begin{aligned}
H^{*}(S) & \rightarrow I^{*}(S) \\
v & \rightarrow V *_{\alpha} u_{W}
\end{aligned}
$$

is an isomorphism.

In the equivariant case, we obtain a Thom isomorphism in equivariant cohomology from the ordinary Thom isomorphism of the disc bundle $\left(E \times B_{\varepsilon}^{+}\right) / G$ over $(E \times S) / G$. This completes the proof of proposition 1 .

\section{Proof of theorem 2}

Let $(X, A)$ be an index pair of the isolated invariant set $S^{\prime}$. In order to find a relation between $I^{*}\left(S^{\prime}\right)$ and $H^{*}\left(S^{\prime}\right)$, consider the compact set $X^{\infty}$ of definition 1 and the commutative diagram

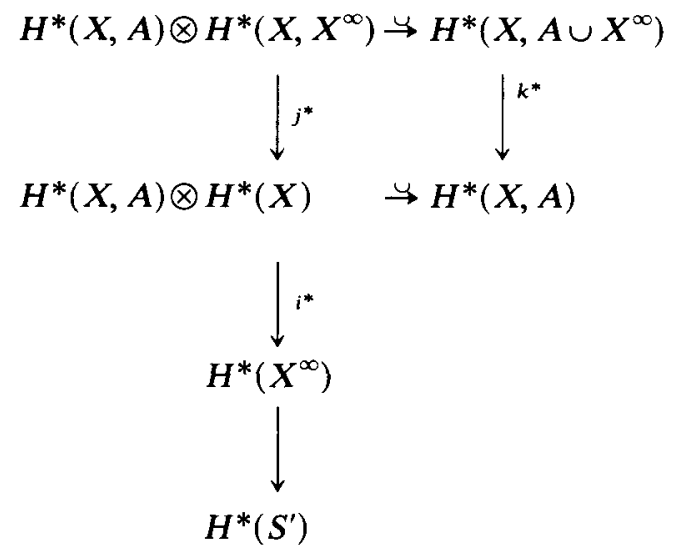

where the homomorphisms $i^{*}, j^{*}$, and $k^{*}$ are induced by inclusions. 
Lemma 5. If $(X, A)$ is an index pair for $S^{\prime}$, then $H^{*}\left(X, A \cup X^{\infty}\right)=0$.

Proof. We apply lemma 2 to $X^{\infty}=\bigcap_{t>0} X^{t}$, see (4.1). Consider the exact sequence of the triple $\left(X, A \cup X^{t}, A\right)$

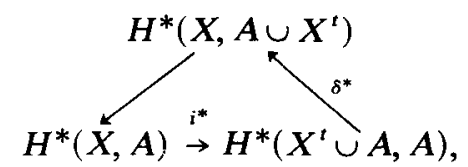

see, for example, $\left[10\right.$, p. 201]. $i^{*}$ is an isomorphism, since in the diagram

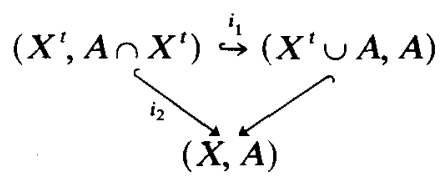

$i_{1}^{*}$ is an isomorphism by excision and $i_{2}^{*}$ is an isomorphism by (4.2). Therefore, $H^{*}\left(X, A \cup X^{t}\right)=0$. This proves the lemma.

We now show that the map (2.8) is injective. In fact, let us assume that $\left(r \mid s_{S^{\prime}}\right)^{*} \psi=0$ for a class $\psi \in H^{*}(S)$. Then by (1) of lemma 4, we have

$$
\left(\left.r\right|_{X^{\infty}}\right)^{*} \psi=i^{*}\left(\left.r\right|_{X}\right)^{*} \psi=0
$$

for some index pair $(X, A)$ of $S^{\prime}$. By exactness of the sequence $\left(i^{*}, j^{*}\right)$, there exists a class $\phi \in H^{*}\left(X, X^{\infty}\right)$ with $j^{*} \phi=\left(\left.r\right|_{X}\right)^{*} \psi$. But then for all $u \in I^{*}\left(S^{\prime}\right)=H^{*}(X, A)$ :

$$
\psi *, u=u \cup\left(\left.r\right|_{X}\right)^{*} \psi=k^{*}(u \cup \phi)=0,
$$

since the group $H^{*}\left(X, A \cup X^{\infty}\right)$ vanishes by lemma 5 . Here, $*_{r}$ denotes the operation of $H^{*}(S)$ on $I^{*}\left(S^{\prime}\right)=H^{*}(X, A)$ defined by the continuous retraction $r: \Gamma \rightarrow S$, see (1.1). Now note that by the hypothesis of theorem 2, the isolated invariant sets $S$ and $S^{\prime}$ are related by continuation. Hence by theorem 1 , the $H^{*}(S)$-module structure of $I^{*}\left(S^{\prime}\right)$ defined by this operation is isomorphic to the $H^{*}(S)$-module structure of $I^{*}(S)$. Since $I^{*}(S)$ is a free $H^{*}(S)$-module by the definition of a $*$-hyperbolic set, the same is true for $I^{*}\left(S^{\prime}\right)$. Consequently, (6.1) holds for every $u \in I^{*}\left(S^{\prime}\right)$, so that $\psi=0$ in $H^{*}(S)$. This completes the proof of theorem 2 .

\section{REFERENCES}

[1] V. Benci. MRC-report, to appear.

[2] C. C. Conley. Isolated Invariant Sets and the Morse Index. CBMS, Regional Conf. Series in Math., vol. 38 (1978).

[3] C. Conley \& E. Zehnder. Morse type index theory for Hamiltonian equations. Comm. Pure and Appl. Math. XXXVII (1984), 207-253.

[4] F. T. Fadell \& P. H. Rabinowitz. Generalized cohomological index theories for Lie group actions with an application to bifurcation questions for Hamiltonian systems. Inv. Math. 45 (1978), 139-174.

[5] N. Fenichel. Persistence and smoothness of invariant manifolds for flows. Ind. Univ. Math. J. 21, No. 3 (1971).

[6] A. Floer. Proof of the Arnold conjecture for surfaces and generalizations for certain Kähler manifolds. To appear in Duke Math. J., vol. 42.

[7] A. Floer \& E. Zehnder. Fixed points results for symplectic maps related to the Arnold-Conjecture. Proceedings of workshop in Dynamical Systems \& Bifurcations, Groningen 16. -19. April 1984. 
[8] S. Helgason. Differential Geometry, Lie groups and Symmetric Spaces. New York, 1978: Academic Press.

[9] M. W. Hirsch, C. C. Pugh \& M. Shub. Invariant Manifolds. Lecture Notes in Math. 583, BerlinHeidelberg-New York 1977: Springer.

[10] E. Spanier. Algebraic Topology. New York 1966: McGraw Hill. 